\title{
The brain-derived neurotrophic factor Val66Met polymorphism increases segregation of structural correlation networks in healthy adult brains
}

\author{
Issei Ueda ${ }^{\text {Corresp., } 1}$, Kazuhiro Takemoto ${ }^{2}$, Keita Watanabe ${ }^{1}$, Koichiro Sugimoto ${ }^{1}$, Atsuko Ikenouchi ${ }^{3}$, Shingo Kakeda \\ ${ }^{1}$, Asuka Katsuki ${ }^{3}$, Reiji Yoshimura ${ }^{3}$, Yukunori Korogi ${ }^{1}$ \\ ${ }^{1}$ Department of Radiology, University of Occupational and Environmental Health, Kitakyusyu, Japan \\ 2 Department of Bioscience and Bioinformatics, Kyushu Institute of Technology, Ilzuka, Japan \\ 3 Department of Psychiatry, University of Occupational and Environmental Health, Kitakyusyu, Japan \\ Corresponding Author: Issei Ueda \\ Email address: i-ueda@med.uoeh-u.ac.jp
}

Background. Although structural correlation network (SCN) analysis is an approach to evaluate brain networks, the neurobiological interpretation of $\mathrm{SCN}$ s is still problematic. Brain-derived neurotrophic factor (BDNF) is well-established as a representative protein related to neuronal differentiation, maturation, and survival. Since a valine-to-methionine substitution at codon 66 of the BDNF gene (BDNF Val66Met single nucleotide polymorphism [SNP]) is well-known to have effects on brain structure and function, we hypothesized that SCNs are affected by the BDNF Val66Met SNP. To gain insight into SCN analysis, we investigated potential differences between BDNF valine (Val) homozygotes and methionine (Met) carriers in the organization of their SCNs derived from inter-regional cortical thickness correlations.

Methods. Forty-nine healthy adult subjects (mean age $=41.1$ years old) were divided into two groups according to their genotype $(\mathrm{n}$ : Val homozygotes $=16$, Met carriers $=33$ ). We obtained regional cortical thickness from their brain T1 weighted images. Based on the inter-regional cortical thickness correlations, we generated SCNs and used graph theoretical measures to assess differences between the two groups in terms of network integration, segregation, and modularity.

Results. The average local efficiency, a measure of network segregation, of BDNF Met carriers' network was significantly higher than that of the Val homozygotes' (permutation p-value $=0.002$ ). Average shortest path lengths (a measure of integration), average local clustering coefficient (another measure of network segregation), small-worldness (a balance between integration and segregation), and modularity (a representative measure for modular architecture) were not significantly different between group (permutation $p$-values $\geqq 0.01$ ).

Discussion and Conclusion. Our results suggest that the BDNF Val66Met polymorphism may potentially influence the pattern of brain regional morphometric (cortical thickness) correlations. Comparing networks derived from inter-regional cortical thickness correlations, Met carrier SCNs have denser connections with neighbors and are more distant from random networks than Val homozygote networks. Thus, it may be necessary to consider potential effects of BDNF gene mutations in SCN analyses. This is the first study to demonstrate a difference between Val homozygotes and Met carriers in brain SCNs. 
1 Title

2 The brain-derived neurotrophic factor Val66Met

3 polymorphism increases segregation of structural

4 correlation networks in healthy adult brains

5 Short Title

6 BDNF Val66Met affects structural network

7

8 Authors

9 Issei Ueda ${ }^{1}$, Kazuhiro Takemoto ${ }^{2}$, Keita Watanabe ${ }^{1}$, Koichiro Sugimoto $^{1}$, Atsuko Ikenouchi ${ }^{3}$,

10 Shingo Kakeda ${ }^{1}$, Asuka Katsuki ${ }^{3}$, Reiji Yoshimura ${ }^{3}$, Yukunori Korogi ${ }^{1}$

11

$12{ }^{1}$ Department of Radiology, University of Occupational and Environmental Health, Kitakyusyu,

13 Fukuoka, Japan

$14{ }^{2}$ Department of Bioscience and Bioinformatics, Kyushu Institute of Technology, Iizuka,

15 Fukuoka, Japan

$16{ }^{3}$ Department of Psychiatry, University of Occupational and Environmental Health, Kitakyusyu,

17 Fukuoka, Japan

18

19 Corresponding Author:

20 Issei Ueda ${ }^{1}$ 
21 1-1 Iseigaoka, Yahatanishi-ku, Kitakyushu, Fukuoka, 807-8555, Japan.

22 Email address: i-ueda@med.uoeh-u.ac.jp

23

24 Abstract

25

26

27

28

29

30

31

32

33

34

35

36

37

Background. Although structural correlation network ( $\mathrm{SCN}$ ) analysis is an approach to evaluate brain networks, the neurobiological interpretation of SCNs is still problematic. Brain-derived neurotrophic factor (BDNF) is well-established as a representative protein related to neuronal differentiation, maturation, and survival. Since a valine-to-methionine substitution at codon 66 of the BDNF gene (BDNF Val66Met single nucleotide polymorphism [SNP]) is well-known to have effects on brain structure and function, we hypothesized that SCNs are affected by the BDNF Val66Met SNP. To gain insight into SCN analysis, we investigated potential differences between BDNF valine (Val) homozygotes and methionine (Met) carriers in the organization of their SCNs derived from inter-regional cortical thickness correlations.

Methods. Forty-nine healthy adult subjects (mean age $=41.1$ years old) were divided into two groups according to their genotype ( $\mathrm{n}$ : Val homozygotes $=16$, Met carriers $=33$ ). We obtained regional cortical thickness from their brain T1 weighted images. Based on the inter-regional cortical thickness correlations, we generated SCNs and used graph theoretical measures to assess differences between the two groups in terms of network integration, segregation, and modularity.

Results. The average local efficiency, a measure of network segregation, of BDNF Met carriers' network was significantly higher than that of the Val homozygotes' (permutation p-value $=$ 0.002). Average shortest path lengths (a measure of integration), average local clustering coefficient (another measure of network segregation), small-worldness (a balance between integration and segregation), and modularity (a representative measure for modular architecture) were not significantly different between group (permutation p-values $\geqq 0.01$ ).

Discussion and Conclusion. Our results suggest that the BDNF Val66Met polymorphism may potentially influence the pattern of brain regional morphometric (cortical thickness) correlations. 
50 Comparing networks derived from inter-regional cortical thickness correlations, Met carrier

51 SCNs have denser connections with neighbors and are more distant from random networks than

52 Val homozygote networks. Thus, it may be necessary to consider potential effects of BDNF

53 gene mutations in SCN analyses. This is the first study to demonstrate a difference between Val

54 homozygotes and Met carriers in brain SCNs.

56

57

58

The brain is a complex organ segregated functionally into local areas that differ in their anatomy, and these areas are highly integrated during perception and behavior (Tononi, Sporns \& Edelman, 1994; Bullmore \& Sporns, 2009; Fornito, Zalesky \& Breakspear, 2015). Since the mid-2000s, researchers have conducted analyses from the perspective of anatomical or functional connectivity to understand the neural basis of normal or abnormal brain function (Sporns, Tononi \& Kötter, 2005; Bullmore \& Sporns, 2009, 2012). The current representative methods for analyses of functional networks include correlations of regional functional magnetic resonance imaging (fMRI) or electroencephalography/magnetoencephalography signals (Evans, 2013), To analyses of anatomical connectivity, structural network analyses based on a streamline network derived from diffusion-weighted imaging (DWI) and a structural correlation network (SCN) derived from structural magnetic resonance imaging (MRI) are typically adopted (Evans, 2013). Research using these connectivity analysis methods is gradually revealing insight into the neural basis of human brain functions and neurological/psychopathological diseases. Such breakthroughs have not been possible with traditional approaches that focused on discrete brain regions (Fornito, Zalesky \& Breakspear, 2015).

SCN analysis is an approach to evaluate brain networks in vivo. The basic idea of SCN analysis is simple: a single morphological feature, such as cortical thickness or gray matter volume, is measured in each region in multiple subject images, and the correlation of estimates for that feature between each brain region is calculated for each pair of all possible regions (Evans 2013). Analysis of brain networks based on structural correlation is expected to be more reproducible than network analyses based on fMRI or DWI because structural correlation analysis reconstructs the entire-brain network using simpler measurements and calculations. 
79 SCN analyses have previously been used to understand brain function (Tuladhar et al. 2015;

80 Chen et al. 2008), the basis of neuropsychiatric disorders (Bernhardt et al. 2008; Bassett et al.

81 2008; Balardin et al. 2015; He et al. 2008; Mueller et al. 2015), and characteristics of brain

82 development (Khundrakpam et al. 2013; Váša et al. 2018; Raznahan et al. 2011). However,

83 neurobiological interpretation of SCNs is still problematic. The basis of SCNs is presumed to

84 involve similarities in terms of cell structure, gene expression, and function, as well as neuronal

85 connectivity (Alexander-Bloch et al. 2013; Evans 2013), but the source of this connectivity is

86 unclear. Therefore, it is important to gain further knowledge regarding SCN analyses given that

87 it is one of the more promising approaches to understand the brain.

88

89

90

Brain areas are highly connected through nerve fibers (axons) of nerve cells (neurons). Brain-derived neurotrophic factor (BDNF) is well-established as a representative protein related to neuronal differentiation, maturation, and survival (Acheson et al., 1995; Huang \& Reichardt, 2001; Binder \& Scharfman, 2004). The human BDNF gene is located on chromosome 11, region p13-14 and it spans $\sim 70 \mathrm{~kb}$. The gene has a complex structure consisting of 11 exons in the 5' end and nine functional promoters (Cattaneo et al., 2016). The human BDNF gene frequently carries a no conserved single nucleotide polymorphism (SNP) that results in a valine-tomethionine substitution at codon 66 (Val66Met). This SNP does not affect BDNF signaling but is reported to cause a deficit in cellular distribution and dysregulated BDNF secretion in neurons (Egan et al., 2003; Kuczewski, Porcher \& Gaiarsa, 2010).

Decreases in activity-dependent release of BDNF are related to the structure and function of the developing brain (Bath \& Lee, 2006). Several studies have reported the effects of the BDNF Val66Met SNP on gray or white matter structural alterations (Pezawas et al., 2004; Montag et al., 2009; Chiang et al., 2011; Tost et al., 2013; Huang et al., 2014), and a number of reports show that the BDNF Val66Met SNP affects brain functions as reviewed in (Toh et al. 2018). Additionally, given that it is accompanied by brain neural circuitry changes, the BDNF Val66Met SNP is thought to be related to the psychopathology of several neuropsychiatric disorders including major depression (Frodl et al., 2007), schizophrenia (Ho et al., 2006), and others (Harrisberger et al., 2015). Furthermore, the BDNF protein is related to brain development and maturation because BDNF influences neuronal proliferation, differentiation and survival of neurons, as well as neural morphology and function, synaptic revisions, axonal maintenance, and

Peer] reviewing PDF | (2020:02:45587:1:0:NEW 23 Jun 2020) 
109 neuroplasticity throughout life (Egan et al., 2003; Frielingsdorf et al., 2010; Jasińska et al., 2017).

110 Several recent studies describe the effects of the Val66Met SNP on structural and

111 functional networks in the brain (Ziegler et al., 2013; Park et al., 2017; Schweiger et al., 2019;

112 Franzmeier et al., 2019). Also, recent cortical correlation network studies on brain

113 developmental changes in adolescence showed that brain integration, segregation and modularity

114 change as a result of the developmental process of pruning combined with consolidation of

115 surviving connections (Khundrakpam et al., 2013; Váša et al., 2018). Based on these reports, we

116 hypothesized that the BDNF SNP affects segregation, integration, and modular architectures of

117 SCN.

118 Thus, the purpose of this study was to gain new insights regarding SCN analysis. Given

119 that the BDNF Val66Met SNP is known to affect brain structure and function, we hypothesized

120 that this SNP also affects brain SCNs. We examined whether there are differences between the

121 brains of BDNF methionine (Met) carriers and valine (Val) homozygotes in terms of SCNs.

\section{Materials and Methods}

124 To investigate the effects of BDNF gene mutation on brain SCNs, we created a Val 125 homozygote structural network and a Met carrier network based on inter-regional cortical 126 thickness correlations, and we conducted statistical comparisons of graph theoretical measures 127 using permutation simulations. See Fig. 1 for an overview of the analysis in this study.

\section{Participants}

The Local Ethics Committee of the University of Occupational and Environmental

130 Health, Kitakyushu, Japan (UOEH) granted Ethical approval to carry out the study (Ethical 131 Application Ref: 第セ H25-13 号). A copy of the IRB approval documentation and the blank 132 consent form are provided as supplemental files. All participants were informed about the 133 purpose of the study, and written informed consents were obtained from all subjects via the 134 forms. 
135 Forty-nine subjects of Japanese origin were included in the study (mean age 41.1 years, 136 standard deviation $=11.3$ years; range 20-65 years; male:female $=36: 13$; see Table 1 ).

137 Most of the participants were recruited from the psychiatric or radiology departments of

138 UOEH. None of the participants reported any neurological and/or psychopathological disorder

139 (e.g., depression, attention deficit/hyperactivity disorder, and/or schizophrenia) in a simple

140 questionnaire enquiring about their lifetime history of such diseases. All of the participants were

141 right handed. We also asked for the participants' number of years of education; this variable 142 might help to better compare the results of future studies investigating the effect of the BDNF

143 Val66Met polymorphism on the structure of the brain. However, as nearly all participants were 144 medical doctors, no differences in education were observed. Because of the recruitment 145 procedure used (medical doctors, characterized by a higher percentage of male doctors), males 146 were over-represented in the investigated sample. All participants were of East Asian ethnicity.

After undergoing MRI scans, two radiologists with expertise in neuroradiology evaluated the images and confirmed that there were no abnormalities in the participants' brain. Then each participant provided a blood sample for genotyping the BDNF Val66Met polymorphism.

\section{Genetic Analysis}

DNA was extracted from the blood samples of each of the 49 participants according to standard laboratory protocols. DNA was isolated from peripheral blood mononuclear cells using the QIAamp (R) DNA Mini-Kit (QIAGEN, Tokyo, Japan). Genotyping was carried out with a polymerase chain reaction single nucleotide polymorphism (SNP) genotyping system using a

156 BigDye Terminator v3.1 Cycle Sequencing Kit (Life Technologies Japan, Tokyo, Japan). The DNA was read using a BMG Applied Biosystem 3730xI DNA Analyzer (Life Technologies Japan, Tokyo, Japan). We used a forward primer (ATGAAGGCTGCCCCCATGAAA) and a reverse primer (TGACTACTGAGCATCACCCTG) for the BDNF Val66Met polymorphism.

160 The participants were either homozygous for the Val allele (Val/Val genotype), heterozygous 161 (Val/Met genotype), or homozygous for the Met allele (Met/Met genotype). 
163 the Met/Met genotype is less prevalent in Japan (about 15.9\% (Shimizu, Hashimoto \& Iyo, 164 2004)), participants were grouped according to the occurrence of the Met allele, which resulted

165 in two independent groups: Val homozygotes (Val/Val genotype) and Met carriers (Val/Met and 166 Met/Met genotypes).

167 Analyses of Demographic and Genotyping Data

168

169

170

171

172

173

174

175

176

177

178

179

180

181

182

183

184

185

186

187

188

To assess the demographics and characteristics of participants, a nonparametric rank test (Wilcoxon-Mann-Whitney test) was applied to compare the differences in age and years of education among the subjects that were either Met carriers or Val homozygotes. Fisher's exact test was used to evaluate the difference in gender among the groups. To assess the existence of any sampling bias within our subjects, we confirmed that the genotype distributions did not deviate from the expected frequency under Hardy-Weinberg equilibrium and tested the expected Japanese genotype/allele frequencies (Shimizu, Hashimoto \& Iyo, 2004) of the BDNF gene polymorphism using chi-square tests.

These statistical analyses were performed using R software (version 3.4.1, R Foundation for Statistical Computing, Vienna, Austria). The level of significance was set to $p<0.05$ for all demographic experiments.

\section{MRI Acquisition}

MRI scans were performed on a 3.0-Tesla scanner (Signa EXCITE 3T; GE Healthcare, Milwaukee, WI, USA) using a dedicated eight-channel phased-array coil (USA Instruments Aurora, OH, USA). Three-dimensional fast spoiled gradient recalled acquisition with steady state (3D-FSPGR) images of the whole head were obtained with the following parameters:10/4.1/700 (repetition time ms/echo time ms/inversion time), a flip angle of 10, a 24- $\mathrm{cm}$ field of view and 1.2 -mm-thick sections with $0.47 \times 0.47 \times 0.6 \mathrm{~mm}^{3}$ resolution. In addition, diffusion tensor images (DTIs) were also obtained assuming other hypothesis study. Although these DTIs were not used for this study's analysis, the images were used to check whether the subject brains had any abnormalities. 
189 Regional Cortical Thickness Measurement

190

191

192

193

194

195

196

197

198

199

200

201

202

203

204

205

206

207

208

209

210

211

212

213

215

214 We examined the statistical differences in the cortical thickness of each region between 2162.

We used the FreeSurfer software package (version 6.0, http://sufer.nmr.mgh.harvard.edu) for cortical surface reconstruction and cortical thickness estimation. Because detailed procedures for using FreeSurfer have previously been by authors such as (Dale, Fischl \& Sereno, 1999; Fischl \& Dale, 2000; Desikan et al., 2006; Balardin et al., 2015), we describe them here only briefly. The FreeSurfer processing stream consists of the co-registration of the subject T1 image to an atlas for bias correction and intensity normalization, and for brain extraction/removal of non-brain tissue. The cortical boundaries between the gray and white matter, and between the gray matter and cerebrospinal fluid, were tracked, tessellated and smoothed to produce a surface mesh. Topology correction and surface deformation were applied and cortical thickness was estimated by considering the closest distance from the gray/white matter boundary to the gray matter/cerebrospinal fluid boundary at each vertex on the tessellated surface. The cortical surface of each hemisphere was then parcellated based on gyral and sulcal landmarks according to the Desikan-Killiany atlas (Desikan et al., 2006). After FreeSurfer processing, 34 separated cerebral regions for each hemisphere (a total of 68 regions) were identified. The 68 brain regions' names and the abbreviations for these names are summarized in Supplementary Table 1.

Because a research member who was an expert in using FreeSurfer processing visually checked the final FreeSurfer outputs for accuracy or processing failures in a systematic manner, and all of the subjects' outputs were confirmed to be correctly labeled and measured. Thus, we obtained 49 regional thickness values for each of the 68 brain regions.

Prior to the construction of structural correlation networks, FreeSurfer cortical thicknesses were corrected using the following steps: first, in order to remove the effects of age, gender, and an age-gender interaction, linear regression model analyses was conducted at every region with the best model based on the Akaike information criterion (AIC). Next, the residuals of the linear models were substituted for the raw cortical thickness values. 15 the two groups in a supplementary analysis. The results are summarized in Supplementary Table 


\section{Network Construction}

218 For network construction, we first defined anatomical connections as statistical

219 associations in cortical thickness between the brain regions in order to characterize human brain

220 networks. Such a morphometry-based connection concept has been described in many previous

221 studies (Worsley et al., 2005; Lerch et al., 2006; He, Chen \& Evans, 2007; Váša et al., 2018).

222 We evaluated statistical similarities in cortical thickness between all region pairs using

223 Spearman correlation coefficients ( $r$ ) across subjects in each group. The correlation coefficient $r$

224 ranges in value from 1 (positive correlation) to -1 (negative correlation), and the closer to 1 or -1

225 the more significant the correlation. However, to avoid the complication of statistical feature

226 descriptions in subsequent graph theoretical analyses, we converted $r$ values into absolute values.

227 (As a supplementary analysis, we conducted a negative-edge-removed version analysis to check

228 if the results change when edges with negative weight were removed. Please refer to

229 Supplementary Analysis 1). In addition, we conducted edge selection based on Random Matrix

230 Theory (RMT) as inspired by Deng et al. (Deng et al. 2012). RMT was first proposed by Winger

231 and Dyson as a powerful method to identify and model phase transitions associated with disorder

232 and noise in statistical physics and material science (Wigner 1967). The RMT method is able to

233 automatically identify a threshold to construct a mathematically meaningful network from a

234 correlation matrix. We used a RMT-based approach to conduct threshold scanning. Correlation

235 coefficients greater than the threshold were retained, and the remaining coefficients were set to

236 zero.

237 Thus, two inter-regional correlation matrices $(N \times N$, where $N$ is the number of brain

238 regions, here $N=68$ ) consisting of correlations between every pair of brain regions were

239 acquired using the 16 subjects' brains included in the Val homozygote group and 33 subjects'

240 brains included in the Met carrier group respectively.

241 To perform graph theoretical analyses, we used the anatomical inter-regional correlation

242 matrices obtained above as the adjacency matrices of undirected weighted graphs. Nodes and

243 edges represented brain regions and connections between the regions, respectively. The edges

244 weight $w_{i j}$ (an element of the adjacency matrix) corresponds to $|r|$ between brain regions $i$ and $j$;

245 however, they were set as $1 /|r|$ when calculating shortest path lengths. 
246

247

248

249

250

251

252

253

254

255

256

257

258

259

260

261

262

263

264

265

266

267

268

269

270

271

272

\section{Network Comparison}

For network comparison, we first visualized the comparisons between pairs of networks using heat maps and circular graphs. The heat maps were made with R-package ggplot2 (version 3.2.1; ggplot2.tidyverse.org). The circular graphs were made using the Python software program (version 3.6.8; www.python.org) and the MNE-Python (v0.19) package (mne.tools/stable/index.html) (Gramfort et al., 2013)).

Next, we compared the networks using network analysis. According to our hypothesis on the BDNF SNP's effects on integration, segregation, and modularity, we examined the differences in average shortest path length (avgSPL), average local clustering coefficient $(\operatorname{avg} L C C)$, average local efficiency $(\operatorname{avg} L E F F)$, small-worldness $(S W)$, and modularity $(Q)$ inspired by previous studies (Latora \& Marchiori, 2001; Rubinov \& Sporns, 2010, 2011). $\operatorname{avgSPL}$ is a representative measure for integration, and $\operatorname{avgLCC}$ and $\operatorname{avg} L E F F$ are well used measures of segregation. $S W$ is a balance between $\operatorname{avgSPL}$ and $\operatorname{avgLCC}$, and $Q$ is a representative measure for the modular architecture of a network. We computed these network measures using R software version 3.3.1 (www.R-project.org) and the R-package igraph version 1.0.1 (igraph.org).

avgSPL is the average shortest path length among all reachable node pairs and was calculated using the function shortest.path in the igraph package.

$\operatorname{avgLCC}$ indicates the average strength of local-scale connectivity and is defined as $\frac{1}{N}$ $\sum_{i=1}^{N} C_{i}^{w}$, where $C_{i}^{w}$ is a weighted version of the nodal clustering coefficient defined as $C_{i}^{w}=$ $\frac{1}{s_{i}\left(k_{i}-1\right)} \sum_{j, h \in n n(i), i \neq j \neq h} \frac{w_{i j}+w_{i h}}{2} \operatorname{sign}\left(w_{i j} w_{i h} w_{j h}\right)$. Here $n n(i)$ is the set of the nearest neighbors of node $i . k_{i}$ is the degree of node $i ; k_{i}=\sum_{j=1}^{N} \operatorname{sign}\left(w_{i j}\right)$, and $\operatorname{sign}(x)$ is the sign function. $C_{i}^{w}$ was computed using the function transitivity in the igraph package.

avgLEFF indicates average information efficiency among nearest neighbor (i.e., at local level), and in is defined as $\frac{1}{n} \sum_{i \in N} E_{l o c}^{w}(i)$, where $E_{l o c}^{w}(i)$ is the local efficiency of node $i$ defined as $E_{l o c}^{w}(i)=\frac{\Sigma\left[d_{j h}^{w}\right]^{-1}}{k_{i}\left(k_{i}-1\right)}$, where $k_{i}$ is degree of node $i, n n(i)$ is the set of the nearest neighbors of node $i$, and $d_{i j}^{w}$ is the shortest path length between $i$ and $j$ in a weighted network. 

property in real-world networked systems (Watts \& Strogatz, 1998). The small-world property indicates that all node pairs in a network were reachable by a short distance (i.e., the distance

276 expected from random networks), even though the network is divided into highly interconnected clusters (i.e., the network is far from a random network). Specifically, SW was calculated based on the avgSPL and $\operatorname{avgLCC}$ in the actual and randomized networks: (i.e., $\operatorname{avg} L C C$ or $\operatorname{avgSPL}$ ) in the actual networks, and $X_{\text {rand }}$ is the average $X$ obtained from 100 randomized networks. For an actual network, the randomized networks were generated by permutation of the edge weights in the original network. whether a particular division of a network into communities is strong or weak division (Newman, 2004a,b). We considered a weighted version of the $Q$-value (Fortunato, 2010). The $Q$ value is defined as the fraction of edge weights that lie within, rather than between, modules relative to that expected by chance as follows:

$$
Q=\frac{1}{2 W} \sum_{i j}\left(w_{i j}-\frac{s_{i} s_{j}}{2 W}\right) \delta\left(c_{i}, c_{j}\right),
$$

where $W$ is the sum of the weights of all edges, and $\delta\left(c_{i}, c_{j}\right)=1$ if nodes $i$ and $j$ belong to the same module but 0 otherwise. A network with a higher $Q$ indicates a higher modular structure. In

291 the present study, an algorithm based on simulated annealing (Guimerà \& Nunes Amaral, 2005) was used to find the maximum $Q$ in order to avoid the resolution limit problem in community (or module) detection (Fortunato \& Barthélemy, 2007; Fortunato, 2010) wherever possible. The maximum $Q$ was defined as the network modularity of brain networks. We used the netcarto function in the R-package rnetcarto (version 0.2.4) to compute network modularity $Q$.

We did not consider regional network characteristics (i.e. local network measures)

297 because we could not find any report on the BDNF SNP's effects on specific brain regions. To

298 show statistically significant differences in the network measures between Val homozygotes and

299 Met carriers, we calculated type I error rates using the permutation method under the null

300 hypothesis. Details of the statistical method are described in the next section. 
302 Network Measure Comparison with Permutation Method

303 To statistically test the difference in the network measures between the Met carriers'

304 network and the Val homozygotes' network, we considered a permutation test approach for 305 weighted correlation networks (Bassett et al., 2008; Gill, Datta \& Datta, 2010). This approach

306 randomly permutes the regional cortical thickness data sets from the subjects, and contrasts two

307 networks built by partitioning the 49 subjects into two groups. We calculated the network

308 measures from the permuted networks and obtained the approximate p-value by computing the

309 probability that the simulated differences are greater than or equal to the observed difference.

310 That is,

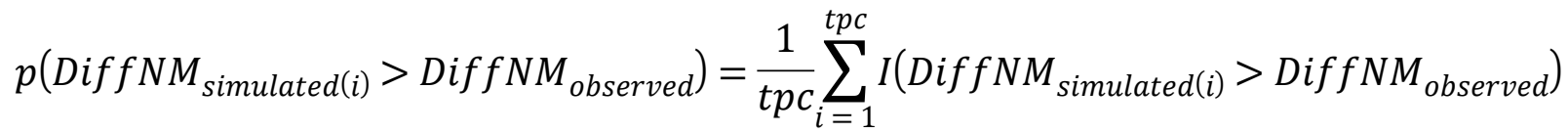

312

$p\left(\right.$ DiffN $\left.M_{\text {simulated }(i)}<\operatorname{DiffN}_{\text {observed }}\right)=\frac{1}{t p c} \sum_{i=1}^{t p c} I\left(\operatorname{DiffN} M_{\text {simulated }(i)}<\operatorname{DiffN} M_{\text {observed }}\right)$

313 , where $p(x)$ is the $\mathrm{p}$-value under the condition $x$. tpc is the total permutation count (in this study,

$314 t p c=1000$ ). DiffNM $M_{\text {simulated }(i)}$ is the difference between the network measures calculated from the

315 permutated Met carriers' network and permutated Val homozygotes' network obtained at $i$ th

316 trial, and Diff $N M_{\text {observed }}$ is the difference in the network measure between the observed Val

317 homozygote and Met carrier networks. The p-values of every network measure were computed

318 simultaneously using the same set of random permutations.

319 Under the null hypothesis the correlation pattern of each region is the same: the

320 hypothesis was tested based on this permutation scheme being confirmed. That is, between the

321 pseudo-two groups generated by permutation simulation, we calculated the network measure

322 differences, and we examine the frequency that each simulated difference is greater (or less) than

323 the observed difference. If the frequency is less than the predetermined significant level, then we

324 reject the null hypothesis that there is no significant difference between the two groups, and we

325 accept the alternative hypothesis that there is a significant difference between them. A one-sided

326 nonparametric permutation test was used to test for global differences of the network measures

327 between the two groups as specified by the hypotheses. The number of the repetition time for the

328 permutation tests was set to 1000 based on the rules of thumb and the load on the computer. We

329 controlled the familywise error rate with the Bonferroni correction; the critical value (alpha) for 
330 an individual test by dividing the familywise error rate $(0.05)$ by the number of tests. Since we

331 conducted five statistical tests respecting global network measures in this study, the critical value

332 for an individual test was $0.05 / 5=0.01$, and we only consider individual tests with p-value $<$

3330.01 to be significant. Statistical analyses were performed by using R software (version 3.6.1, R

334 Foundation for Statistical Computing, Vienna, Austria).

\section{Results}

\section{Demographic and Genotyping Data}

337

338

339

340

341

342

343

344

345

346

347

348

349

350

351

352

353

354

355

Table 1 displays the genotype frequency, gender, age, and years of education in each of the two groups. Age, years of education and gender were not significantly different in the two genotype groups ( $\mathrm{p}$-values $=0.73,0.996$ and 0.508 respectively).

Furthermore, the genotype distribution of the Val66Met polymorphism in our healthy Japanese cohort comprised $16 \mathrm{Val} / \mathrm{Val}$ homozygotes (32.7\%), $28 \mathrm{Val} / \mathrm{Met}$ heterozygotes (57.1\%), and $5 \mathrm{Met} /$ Met homozygous subjects $(10.2 \%)$. The distribution of genotypes did not differ from the frequencies expected from Hardy-Weinberg equilibrium $\left(\chi^{2}=2.03, \mathrm{df}=1, \mathrm{p}\right.$-value $\left.=0.363\right)$. The allele and genotype frequencies are consistent with those reported previously for Japanese individuals (Shimizu, Hashimoto \& Iyo, 2004) (allele: $\chi^{2}=0.109$, df $=1, p$-value $=0.741$; genotype: $\chi^{2}=1.478, \mathrm{df}=2$, $\mathrm{p}$-value $=0.478$ ).

\section{Network Comparison}

First, to understand the overviews of the structural correlation networks of both groups, we constructed heat maps (Fig. 2). When the heat maps of the two groups were compared, a grid pattern was more clearly perceived in the Met carrier heat map than in the Val homozygote heat map. In a heat map of the brain network, the grids represent sub-divided brain regions (i.e., modules). From these grid patterns, we observed that the Met carrier brain network had clearer module architecture than the Val homozygote brain network. When we focused on the colors in each heat map, the Met carriers' map had more red areas whereas the Val homozygote heat map had more green areas. This observation suggested that the Val homozygote networks consisted of 
356

357

358

359

360

361

362

363

364

365

366

367

368

369

370

371

372

373

374

375

376

377

378

379

380

381

382

383

middle connections whereas the Met carrier networks had more connections with high strength.

Next, we constructed circular graphs using the 100 strongest connections (Fig. 3). In both groups, each brain region had a strong correlation with the counterpart of the region which is represented by the white lines straddling the midline of the each circular graph. This finding seemed to reflect the anatomical left-right symmetry of the cerebrum. In addition, the Met carrier network had fewer brain regions related to the 100 strongest connections than the Val homozygote network. When we focused on the color of the edges, there is no apparent difference between the Val homozygote graph and Met carrier graph.

The results of the statistical comparisons of network measures related to integration, segregation (i.e., $\operatorname{avgSPL}, \operatorname{avgLCC}$, $\operatorname{vvgLEFF}$, and $S W$ ), and modular architecture (i.e. $Q$ ) are given in Table 2. The avgLEFF of the Met carriers' network was larger than that of the Val homozygotes' network, and the statistical difference between the avgLEFF of the two networks was significant (p-value $<0.01$ ). Although the Met carriers network's avgLCC and $S W$ were larger, and $\operatorname{avgSPL}$ and $Q$ were smaller, than those of the Val homozygote network, there were no significant differences between the two groups in regard to these measures ( $p$-value $\geqq 0.01$ ). The permutation distributions and permutation $p$-values for these network measures are shown in Fig. 4.

\section{Discussion}

We examined whether the BDNF Val66Met SNP makes any difference to the brain structural network, and we found that this SNP influences the correlation patterns of the regional cortical thickness; the $\operatorname{avgLEFF}$ (a measure of network segregation) of the BDNF Met carriers' network was significantly higher than that of the Val homozygotes'. avgLEFF is the average of nodal local efficiency of all nodes in a network. The nodal local efficiency is a measure of segregation based on calculated from the average inverse shortest path length to all neighbor nodes (Rubinov and Sporns 2010; Latora and Marchiori 2001), and the average of all nodes (i.e., avgLEFF) can be regarded as a measure of segregation of a whole network. Thus a network with higher $\operatorname{avgLEFF}$ has greater local efficiency and is thought to have less random topology (Fornito, Zalesky \& Breakspear, 2013). Therefore, our data suggest that the Met carriers' 
384 structural correlation networks have denser connections with neighbors and are more distant

385 from random networks than are the Val homozygotes' networks.

386

387

388

389

390

391

392

393

394

395

396

397

398

399

400

401

402

403

404

405

406

407

408

409

410

411

412

413

In contrast to the $\operatorname{avgLEFF}$, we found no significant differences in the avgLCC, $\operatorname{avgSPL\text {,}}$ and $S W$ of Met carriers' and Val homozygotes' network. $\operatorname{avgLCC}$ is the average of nodal clustering coefficients of all nodes in a network, and the nodal clustering coefficient is a measure of segregation based on the number of triangles around an individual node (Watts and Strogatz 1998). avgSPL is the most commonly used measure of network integration (Rubinov \& Sporns, 2010); the calculation formula reflects the average distance between all pairs of nodes in the network. $S W$ is a network property that quantify the balance between integration and segregation (Lord et al., 2017). Our results for these measures suggest that brain structural correlation networks maintain a balance between segregation and integration even when they have the BDNF Val66Met SNP. However, considering the relatively low p-values of the $\operatorname{avg} L C C$ and $S W$ differences, there remains some possibility that the degree of the balance between network segregation and integration would differ if Val homozygote and Met Carrier structural correlation networks were compared using a greater sample size.

We also found no significant difference in modularity $(Q)$ between the Met carriers' and Val homozygotes' networks. Modularity is a measure used to quantify the degree to which the network may be sub-divided into clearly delineated and non-overlapping groups (Newman, 2004a; Rubinov \& Sporns, 2010). Although a heat map comparison gave the impression that the Met carriers' brains had more clear modular architecture, the difference could not be confirmed statistically. Thus, the implied result from the heat map was not reflected in the modularity value we calculated. Given the modularity formula used in this study, the lack of a difference between the modularity values of the two networks only indicates that the strengths of the intra- and intermodule connectivities are almost equal, but it does not indicate that other modular features (e.g. number, member or size) are equal. Thus, the lack of modularity difference we found does not necessarily disagree with our first impression from the heat maps. To examine potential modular differences in more detail, we would need to introduce other concepts such as pre-defined module analysis, which has been conducted in previous studies (Park et al., 2017; Váša et al., 2018), or network analysis using the measurement of well-connected within module and welldistributed between module, which was suggested by Guimera et al. (Guimerà \& Nunes Amaral, 
414 2005).

415 This is the first study to demonstrate a difference between Val homozygotes and Met

416 carriers in brain structural correlation network. However, to our knowledge, two DWI studies

417 have previously examined the effects of the BDNF Val66Met SNP on the brain structural

418 network. Using DWI, Ziegler et al. examined differences in structural brain connectivity between

419 Met carriers and Val homozygotes, and they showed that Met carriers' structural connectivity

420 was greatly increased throughout the forebrain (Ziegler et al., 2013). In addition, Park et al.

421 assessed the effect of the SNP on the network properties and robustness of the structural

422 networks derived from white matter tractography (Park et al., 2017), and they found that the

423 network of the Met carriers group showed higher vulnerability than Val homozygote group to

424 targeted removal of central nodes.

425 Here, we did not compare our results with those of these previous studies because they

426 relied on binary networks constructed based on diffusion tensor images. Moreover, the network

427 features considered in the previous studies differed from those considered in our study. However,

428 the hypothesis stated in Ziegler's study (Ziegler et al., 2013) can be closely related to our results

429 as a plausible explanation for how the BDNF Val66Met SNP causes increased segregation of

430 structural correlation networks. Specifically, Ziegler et al. stated that brain connectivity might

431 eventually be less profoundly shaped by experience in Met carriers than in Val homozygotes if

432 silent axons are relatively less likely to be pruned due to reduced BDNF secretion in the Met

433 carriers. This hypothesis was based on the BDNF Val66Met SNP being known to cause a

434 reduction in activity-dependent BDNF secretion (Egan et al., 2003), as well BDNF studies

435 reporting involvement in long-term potentiation and synaptic plasticity (Patterson et al., 1996)

436 and axonal pruning and maintenance triggering the elimination of synaptically silent axonal

437 terminal arbors (Cao et al., 2007; Singh et al., 2008). The mechanism hypothesized by Ziegler et

438 al. could explain our finding that Met carriers' brains have greater avgLEFF. Perhaps the BDNF

439 Val66Met SNP leads to a reduction in pruning and maintenance of neurons, and the regional

440 connectivities in Met carriers' brains are less enhanced by experience than those in Val

441 homozygotes' brains. As a result, Met carriers' brains develop denser connections among

442 neighboring regions and form a highly clustered network compared to that in Val homozygote

443 brains. However, despite this hypothesis, the detailed mechanism that underlies the BDNF 
444 Val66met SNP's effects on brain structural networks cannot be derived from our data; further 445 studies are therefore required.

446 When utilizing a SCN analysis, researchers have to select appropriate parameters 447 according to the individual study. Although the methodological concept of SCN is relatively 448 simple, there are many parameters that may affect the results of the analysis. These parameters 449 include: definitions of nodes and edges, a measure for quantifying correlations, a method for 450 setting thresholds, a presence or absence of binarization processing, focus of network measures, 451 and other examples. These technical aspects of SCN need to be further examined in future 452 studies. In the current study, for example, the absolute value of the correlation was defined as the 453 weight of each edge based on previous studies (Balardin et al. 2015; He et al. 2008). However, 454 the biological basis of the correlation between two regions is still not clear, and it is difficult to 455 determine a better way to treat the negative correlation. We performed a supplementary analysis 456 (Supplementary Analysis 1) to confirm that our results were not distorted by treating connections 457 with opposite (i.e., negative) directions as equivalent to connections with the same (i.e., positive) 458 direction. Supplementary Analysis 1 shows results following pre-processing to remove the 459 negative correlation. These results were similar to the main analysis and suggest that treating 460 negative correlations the same as positive correlations did not affect the conclusions of this study. 461 Regarding network measures, for another example, we only focused on five network measures 462 (avgSPL, avgLCC, avgLEFF, $S W$, and $Q$ ), but if we had also focused on other measures such as 463 vulnerability or centrality, we may have captured other differences between the two groups. had almost similar backgrounds, and we statistically confirmed that there were no differences 466 between the groups in age, gender and years of education, there is a possibility that the difference 467 we found between Val homozygotes and Met carriers derives from another unknown variable. 468 Thus, a larger sample size would be needed to remove potential effects due to age, gender, 469 cognitive function, and other individual characteristics. In addition, our result should be 470 validated through comparisons of inter-regional correlations in terms of cortical cytoarchitectural 471 or gene expression pattern, comparisons between networks derived from DWI or fMRI. The 472 comparison composition for the current study was limited. Following the related previous studies 473 (Ziegler et al. 2013; Park et al. 2017), we compared SCNs between groups under the composition 
474 of Val homozygotes versus Met carriers in this study. However, given reports that the Val66Met 475 SNP affects the regulation of BDNF secretion (Egan et al. 2003; Kuczewski et al. 2010) and that 476 there were differences in brain structures compared between the three groups (Frode et al. 2014), 477 it is possible that the three groups (i.e., Val/Val, Val/Met, and Met/Met) have different network 478 features. Ideally, we should have extended the analysis to the three-group comparison, but due to 479 a sample size imbalance between the three groups in our study, we did not perform such an 480 analysis. Furthermore, a SCN can only be used for group analyses because it is created from 481 group data and not individual data. This practical limitation hinders the application of results 482 from SCN analyses to directly analyze individual brain networks. However, SCN analyses do 483 have the advantage of simplicity compared to analyses of networks derived from DWI or fMRI. 484 Moreover, there are several studies using methods with multiple morphometric features for 485 generating SCNs in individual subjects (Seidlitz et al. 2018; Li et al. 2017). Finally, while it 486 would be beneficial to evaluate the statistical power and effect size, approaches for comparison 487 of SCNs are not currently established, but in the future bootstrap approaches may be useful. 488 However, this limitation poses little problem given that numerical simulations using synthetic 489 data and previous application studies (Luo et al., 2006; Deng et al., 2012) confirm the validity of 490 the RMT-based method.

491

492

493

494

495

496

497 498 499 500

\section{Conclusion}

Given that the brain is a network, methods involving network analyses are expected to be able to evaluate features that cannot be captured by simply comparing individual regions to each other. SCN analysis is one methods to evaluate the brain as a network. The results of our study suggest that the BDNF Val66Met SNP affects overall segregation in SCNs. Thus, it may be necessary to consider the effect of BDNF gene mutations in future analyses using SCNs. Overall, our results provide further knowledge regarding the analysis of networks derived from brain structural correlations. 
501 References

502 Acheson A., Conover JC., Fandl JP., DeChiara TM., Russell M., Thadani A., Squinto SP.,

503 Yancopoulos GD., Lindsay RM. 1995. A BDNF autocrine loop in adult sensory neurons

504 prevents cell death. Nature 374:450-453.

505 Alexander-Bloch, A., Giedd, J.N. and Bullmore, E. 2013. Imaging structural co-variance

506 between human brain regions. Nature Reviews. Neuroscience 14(5), pp. 322-336.

507 de Araujo CM., Zugman A., Swardfager W., Belangero SIN., Ota VK., Spindola LM.,

508 Hakonarson H., Pellegrino R., Gadelha A., Salum GA. et al. 2018. Effects of the brain-

509 derived neurotropic factor variant Val66Met on cortical structure in late childhood and early

$510 \quad$ adolescence. Journal of Psychiatric Research 98:51-58.

511 Balardin JB., Comfort WE., Daly E., Murphy C., Andrews D., Murphy DGM., Ecker C., MRC

512

513

514

515

516

517

518

519

520

521

522

523

524

525

526

527

528

529

530

531 AIMS Consortium., Sato JR. 2015. Decreased centrality of cortical volume covariance networks in autism spectrum disorders. Journal of Psychiatric Research 69:142-149.

Banner H., Bhat V., Etchamendy N., Joober R., Bohbot VD. 2011. The brain-derived neurotrophic factor Val66Met polymorphism is associated with reduced functional magnetic resonance imaging activity in the hippocampus and increased use of caudate nucleusdependent strategies in a human virtual navigation task. The European Journal of

Neuroscience 33:968-977.

Bassett DS., Bullmore E., Verchinski BA., Mattay VS., Weinberger DR., Meyer-Lindenberg A. 2008. Hierarchical organization of human cortical networks in health and schizophrenia. The Journal of Neuroscience 28:9239-9248.

Bath KG., Lee FS. 2006. Variant BDNF (Val66Met) impact on brain structure and function. Cognitive, Affective \& Behavioral Neuroscience 6:79-85.

Bernhardt, B.C., Worsley, K.J., Besson, P., et al. 2008. Mapping limbic network organization in temporal lobe epilepsy using morphometric correlations: insights on the relation between mesiotemporal connectivity and cortical atrophy. Neuroimage 42(2), pp. 515-524.

Beste C., Schneider D., Epplen JT., Arning L. 2011. The functional BDNF Val66Met polymorphism affects functions of pre-attentive visual sensory memory processes. Neuropharmacology 60:467-471.

Binder DK., Scharfman HE. 2004. Brain-derived neurotrophic factor. Growth Factors 22:123131. 
532 Bullmore E., Sporns O. 2009. Complex brain networks: Graph theoretical analysis of structural

533

534

535

536

537

538

539

540

541

542

543

544

545

546

547

548

549

550

551

552

553

554

555

556

557

558

559

560

561

562 and functional systems. Nature Reviews. Neuroscience 10:186-198.

Bullmore E., Sporns O. 2012. The economy of brain network organization. Nature Reviews. Neuroscience 13:336-349.

Cao L., Dhilla A., Mukai J., Blazeski R., Lodovichi C., Mason CA., Gogos JA. 2007. Genetic modulation of BDNF signaling affects the outcome of axonal competition in vivo. Current Biology 17:911-921.

Cattaneo A., Cattane N., Begni V., Pariante CM., Riva MA. 2016. The human BDNF gene: peripheral gene expression and protein levels as biomarkers for psychiatric disorders. Translational psychiatry 6:e958.

Chen, Z.J., He, Y., Rosa-Neto, P., Germann, J. and Evans, A.C. 2008. Revealing modular architecture of human brain structural networks by using cortical thickness from MRI. Cerebral Cortex 18(10), pp. 2374-2381.

Chiang M-C., Barysheva M., Toga AW., Medland SE., Hansell NK., James MR., McMahon KL., de Zubicaray GI., Martin NG., Wright MJ. et al. 2011. BDNF gene effects on brain circuitry replicated in 455 twins. Neuroimage 55:448-454.

Dale AM., Fischl B., Sereno MI. 1999. Cortical surface-based analysis. I. Segmentation and surface reconstruction. Neuroimage 9:179-194.

Deng, Y., Jiang, Y.-H., Yang, Y., He, Z., Luo, F. and Zhou, J. 2012. Molecular ecological network analyses. BMC Bioinformatics 13, p. 113.

Desikan RS., Ségonne F., Fischl B., Quinn BT., Dickerson BC., Blacker D., Buckner RL., Dale AM., Maguire RP., Hyman BT. et al. 2006. An automated labeling system for subdividing the human cerebral cortex on MRI scans into gyral based regions of interest. Neuroimage 31:968-980.

Egan MF., Kojima M., Callicott JH., Goldberg TE., Kolachana BS., Bertolino A., Zaitsev E., Gold B., Goldman D., Dean M. et al. 2003. The BDNF val66met polymorphism affects activity-dependent secretion of BDNF and human memory and hippocampal function. Cell 112:257-269.

Evans AC. 2013. Networks of anatomical covariance. Neuroimage 80:489-504.

Fischl B., Dale AM. 2000. Measuring the thickness of the human cerebral cortex from magnetic resonance images. Proceedings of the National Academy of Sciences of the United States of 
563

564

565

566

567

568

569

570

571

572

573

574

575

576

577

578

579

580

581

582

583

584

585

586

587

588

589

590

591

592

593

America 97:11050-11055.

Forde, N.J., Ronan, L., Suckling, J., et al. 2014. Structural neuroimaging correlates of allelic variation of the BDNF val66met polymorphism. Neuroimage 90, pp. 280-289.

Fornito A., Zalesky A., Breakspear M. 2013. Graph analysis of the human connectome: promise, progress, and pitfalls. Neuroimage 80:426-444.

Fornito A., Zalesky A., Breakspear M. 2015. The connectomics of brain disorders. Nature Reviews. Neuroscience 16:159-172.

Fortunato S. 2010. Community detection in graphs. Physics Reports 486:75-174.

Fortunato S., Barthélemy M. 2007. Resolution limit in community detection. Proceedings of the National Academy of Sciences of the United States of America 104:36-41.

Franzmeier N., Ren J., Damm A., Monté-Rubio G., Boada M., Ruiz A., Ramirez A., Jessen F., Düzel E., Rodríguez Gómez O. et al. 2019. The BDNFVal66Met SNP modulates the association between beta-amyloid and hippocampal disconnection in Alzheimer's disease.

Molecular Psychiatry.

Freundlieb N., Backhaus W., Brüggemann N., Gerloff C., Klein C., Pinnschmidt HO., Hummel FC. 2015. Differential effects of BDNF val(66)met in repetitive associative learning paradigms. Neurobiology of Learning and Memory 123:11-17.

Frielingsdorf H., Bath KG., Soliman F., Difede J., Casey BJ., Lee FS. 2010. Variant brainderived neurotrophic factor Val66Met endophenotypes: implications for posttraumatic stress disorder. Annals of the New York Academy of Sciences 1208:150-157.

Frodl T., Schüle C., Schmitt G., Born C., Baghai T., Zill P., Bottlender R., Rupprecht R., Bondy B., Reiser M. et al. 2007. Association of the brain-derived neurotrophic factor Val66Met polymorphism with reduced hippocampal volumes in major depression. Archives of General Psychiatry 64:410-416.

Gajewski PD., Hengstler JG., Golka K., Falkenstein M., Beste C. 2011. The Met-allele of the BDNF Val66Met polymorphism enhances task switching in elderly. Neurobiology of Aging 32:2327.e7-19.

Gill R., Datta S., Datta S. 2010. A statistical framework for differential network analysis from microarray data. BMC Bioinformatics 11:95.

Gramfort A., Luessi M., Larson E., Engemann DA., Strohmeier D., Brodbeck C., Goj R., Jas M., Brooks T., Parkkonen L. et al. 2013. MEG and EEG data analysis with MNE-Python. 
594

595

596

597

598

599

600

601

602

603

604

605

606

607

608

609

610

611

612

613

614

615

616

617

618

619

620

621

622

623

624

Frontiers in Neuroscience 7:267.

Guimerà R., Nunes Amaral LA. 2005. Functional cartography of complex metabolic networks. Nature 433:895-900.

Hariri AR., Goldberg TE., Mattay VS., Kolachana BS., Callicott JH., Egan MF., Weinberger DR. 2003. Brain-derived neurotrophic factor val66met polymorphism affects human memoryrelated hippocampal activity and predicts memory performance. The Journal of Neuroscience 23:6690-6694.

Harrisberger F., Smieskova R., Schmidt A., Lenz C., Walter A., Wittfeld K., Grabe HJ., Lang UE., Fusar-Poli P., Borgwardt S. 2015. BDNF Val66Met polymorphism and hippocampal volume in neuropsychiatric disorders: A systematic review and meta-analysis. Neuroscience and Biobehavioral Reviews 55:107-118.

He Y., Chen ZJ., Evans AC. 2007. Small-world anatomical networks in the human brain revealed by cortical thickness from MRI. Cerebral Cortex 17:2407-2419.

He, Y., Chen, Z. and Evans, A. 2008. Structural insights into aberrant topological patterns of large-scale cortical networks in Alzheimer's disease. The Journal of Neuroscience 28(18), pp. 4756-4766.

Ho B-C., Milev P., O’Leary DS., Librant A., Andreasen NC., Wassink TH. 2006. Cognitive and magnetic resonance imaging brain morphometric correlates of brain-derived neurotrophic factor Val66Met gene polymorphism in patients with schizophrenia and healthy volunteers. Archives of General Psychiatry 63:731-740.

Huang C-C., Liu M-E., Chou K-H., Yang AC., Hung C-C., Hong C-J., Tsai S-J., Lin C-P. 2014. Effect of BDNF Val66Met polymorphism on regional white matter hyperintensities and cognitive function in elderly males without dementia. Psychoneuroendocrinology 39:94103.

Huang EJ., Reichardt LF. 2001. Neurotrophins: roles in neuronal development and function. Annual Review of Neuroscience 24:677-736.

Humphries MD., Gurney K., Prescott TJ. 2006. The brainstem reticular formation is a smallworld, not scale-free, network. Proceedings. Biological Sciences / the Royal Society 273:503-511.

Jasińska KK., Molfese PJ., Kornilov SA., Mencl WE., Frost SJ., Lee M., Pugh KR., Grigorenko EL., Landi N. 2016. The BDNF val66met polymorphism influences reading ability and 
625

626

627

628

629

630

631

632

633

634

635

636

637

638

639

640

641

642

643

644

645

646

647

648

649

650

651

652

653

654 655

patterns of neural activation in children. Plos One 11:e0157449.

Jasińska KK., Molfese PJ., Kornilov SA., Mencl WE., Frost SJ., Lee M., Pugh KR., Grigorenko EL., Landi N. 2017. The BDNF Val66Met polymorphism is associated with structural neuroanatomical differences in young children. Behavioural Brain Research 328:48-56.

Khundrakpam BS., Reid A., Brauer J., Carbonell F., Lewis J., Ameis S., Karama S., Lee J., Chen Z., Das S. et al. 2013. Developmental changes in organization of structural brain networks. Cerebral Cortex 23:2072-2085.

Kuczewski N., Porcher C., Gaiarsa J-L. 2010. Activity-dependent dendritic secretion of brainderived neurotrophic factor modulates synaptic plasticity. The European Journal of Neuroscience 32:1239-1244.

Latora V., Marchiori M. 2001. Efficient behavior of small-world networks. Physical Review Letters 87:198701.

Lerch JP., Worsley K., Shaw WP., Greenstein DK., Lenroot RK., Giedd J., Evans AC. 2006. Mapping anatomical correlations across cerebral cortex (MACACC) using cortical thickness from MRI. Neuroimage 31:993-1003.

Li, W., Yang, C., Shi, F., et al. 2017. Construction of Individual Morphological Brain Networks with Multiple Morphometric Features. Frontiers in Neuroanatomy 11, p. 34.

Lord L-D., Stevner AB., Deco G., Kringelbach ML. 2017. Understanding principles of integration and segregation using whole-brain computational connectomics: implications for neuropsychiatric disorders. Philosophical Transactions. Series A, Mathematical, Physical, and Engineering Sciences 375.

Luo, F., Zhong, J., Yang, Y., Scheuermann, R.H. and Zhou, J. 2006. Application of random matrix theory to biological networks. Physics Letters A 357(6), pp. 420-423.

Montag C., Weber B., Fliessbach K., Elger C., Reuter M. 2009. The BDNF Val66Met polymorphism impacts parahippocampal and amygdala volume in healthy humans:

incremental support for a genetic risk factor for depression. Psychological Medicine 39:1831-1839.

Mueller, S.G., Ng, P., Neylan, T., et al. 2015. Evidence for disrupted gray matter structural connectivity in posttraumatic stress disorder. Psychiatry Research 234(2), pp. 194-201.

Newman MEJ. 2004a. Fast algorithm for detecting community structure in networks. Physical Review. E, Statistical, Nonlinear, and Soft Matter Physics 69:066133. 
656 Newman MEJ. 2004b. Analysis of weighted networks. Physical Review. E, Statistical, Nonlinear,

657

658

659

660

661

662

663

664

665

666

667

668

669

670

671

672

673

674

675

676

677

678

679

680

681

682

683

684

685

686 and Soft Matter Physics 70:056131.

Park C-H., Kim J., Namgung E., Lee D-W., Kim GH., Kim M., Kim N., Kim TD., Kim S., Lyoo IK. et al. 2017. The BDNF val66met polymorphism affects the vulnerability of the brain structural network. Frontiers in Human Neuroscience 11:400.

Patterson SL., Abel T., Deuel TA., Martin KC., Rose JC., Kandel ER. 1996. Recombinant BDNF rescues deficits in basal synaptic transmission and hippocampal LTP in BDNF knockout mice. Neuron 16:1137-1145.

Pezawas L., Verchinski BA., Mattay VS., Callicott JH., Kolachana BS., Straub RE., Egan MF., Meyer-Lindenberg A., Weinberger DR. 2004. The brain-derived neurotrophic factor val66met polymorphism and variation in human cortical morphology. The Journal of Neuroscience 24:10099-10102.

Raznahan, A., Lerch, J.P., Lee, N., et al. 2011. Patterns of coordinated anatomical change in human cortical development: a longitudinal neuroimaging study of maturational coupling. Neuron 72(5), pp. 873-884.

Rubinov M., Sporns O. 2010. Complex network measures of brain connectivity: uses and interpretations. Neuroimage 52:1059-1069.

Rubinov M., Sporns O. 2011. Weight-conserving characterization of complex functional brain networks. Neuroimage 56:2068-2079.

Schweiger JI., Bilek E., Schäfer A., Braun U., Moessnang C., Harneit A., Post P., Otto K., Romanczuk-Seiferth N., Erk S. et al. 2019. Effects of BDNF Val66Met genotype and schizophrenia familial risk on a neural functional network for cognitive control in humans. Neuropsychopharmacology 44:590-597.

Seidlitz, J., Váša, F., Shinn, M., et al. 2018. Morphometric Similarity Networks Detect Microscale Cortical Organization and Predict Inter-Individual Cognitive Variation. Neuron 97(1), pp. 231-247.e7.

Shimizu E., Hashimoto K., Iyo M. 2004. Ethnic difference of the BDNF 196G/A (val66met) polymorphism frequencies: the possibility to explain ethnic mental traits. American Journal of Medical Genetics. Part B, Neuropsychiatric Genetics 126B:122-123.

Singh KK., Park KJ., Hong EJ., Kramer BM., Greenberg ME., Kaplan DR., Miller FD. 2008. Developmental axon pruning mediated by BDNF-p75NTR-dependent axon degeneration. 
687

688

689

690

691

692

693

694

695

696

697

698

699

700

701

702

703

704

705

706

707

708

709

710

711

712

713

714

715

Nature Neuroscience 11:649-658.

Soliman F., Glatt CE., Bath KG., Levita L., Jones RM., Pattwell SS., Jing D., Tottenham N., Amso D., Somerville LH. et al. 2010. A genetic variant BDNF polymorphism alters extinction learning in both mouse and human. Science 327:863-866.

Sporns O., Tononi G., Kötter R. 2005. The human connectome: A structural description of the human brain. PLoS Computational Biology 1:e42.

Toh, Y.L., Ng, T., Tan, M., Tan, A. and Chan, A. 2018. Impact of brain-derived neurotrophic factor genetic polymorphism on cognition: A systematic review. Brain and behavior 8(7), p. e01009.

Tononi G., Sporns O., Edelman GM. 1994. A measure for brain complexity: relating functional segregation and integration in the nervous system. Proceedings of the National Academy of Sciences of the United States of America 91:5033-5037.

Tost H., Alam T., Geramita M., Rebsch C., Kolachana B., Dickinson D., Verchinski BA., Lemaitre H., Barnett AS., Trampush JW. et al. 2013. Effects of the BDNF Val66Met polymorphism on white matter microstructure in healthy adults. Neuropsychopharmacology 38:525-532.

Tuladhar, A.M., Reid, A.T., Shumskaya, E., et al. 2015. Relationship between white matter hyperintensities, cortical thickness, and cognition. Stroke 46(2), pp. 425-432.

Váša F., Seidlitz J., Romero-Garcia R., Whitaker KJ., Rosenthal G., Vértes PE., Shinn M., Alexander-Bloch A., Fonagy P., Dolan RJ. et al. 2018. Adolescent tuning of association cortex in human structural brain networks. Cerebral Cortex 28:281-294.

Watts DJ., Strogatz SH. 1998. Collective dynamics of "small-world" networks. Nature 393:440442.

Worsley KJ., Chen J-I., Lerch J., Evans AC. 2005. Comparing functional connectivity via thresholding correlations and singular value decomposition. Philosophical Transactions of the Royal Society of London. Series B, Biological Sciences 360:913-920.

Ziegler E., Foret A., Mascetti L., Muto V., Le Bourdiec-Shaffii A., Stender J., Balteau E., Dideberg V., Bours V., Maquet P. et al. 2013. Altered white matter architecture in BDNF met carriers. Plos One 8:e69290. 


\section{Figure 1}

An overview of the analysis.

First, we created a structural correlation network (SCN) using the FreeSurfer cortical thickness data of 68 brain regions from the BDNF Val homozygotes (Met-) (Observed Met- Network). We also created a SCN of BDNF Met carriers (Met+) using the data from all of the Met carrier subjects in the same manner (Observed Met+ Network). Then, we calculated the average shortest path length (avgSPL), average local clustering coefficient (avgLCC), average local efficient (avgLEFF), small-worldness (SW), and modularity $(Q)$ of each network, and differences of each network measure between the two networks ( $\triangle a v g S P L_{\text {obs }}, \triangle a v g L C C_{\text {obs }}$ $\Delta S W_{\text {obs }}$, and $\Delta Q_{o b s}$ ). Next, we randomly permuted the cortical thickness data sets from the subjects and partitioned the 49 subjects into two groups. We created a surrogate SCN from the 16 subjects of the first group (Pseudo Met- Network), and another surrogate SCN from the 33 subjects of the second group (Pseudo Met+ Network). We calculated avgSPL, avgLCC, avgLEFF, SW, and $Q$ of each pseudo network and subtracted

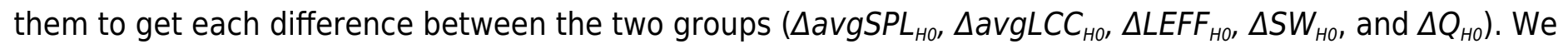
repeated this permutation simulation 1000 times and obtained the distributions of the differences of each measure under the null hypothesis (i.e., the hypothesis that the correlation pattern of each region is the same between the two groups). Finally, we examined significance of the observed difference of each measure by the one-tailed $p$-values calculated as the rate of entries that were greater than (or smaller than) the observed between-group difference in the 1000 times permutations. 


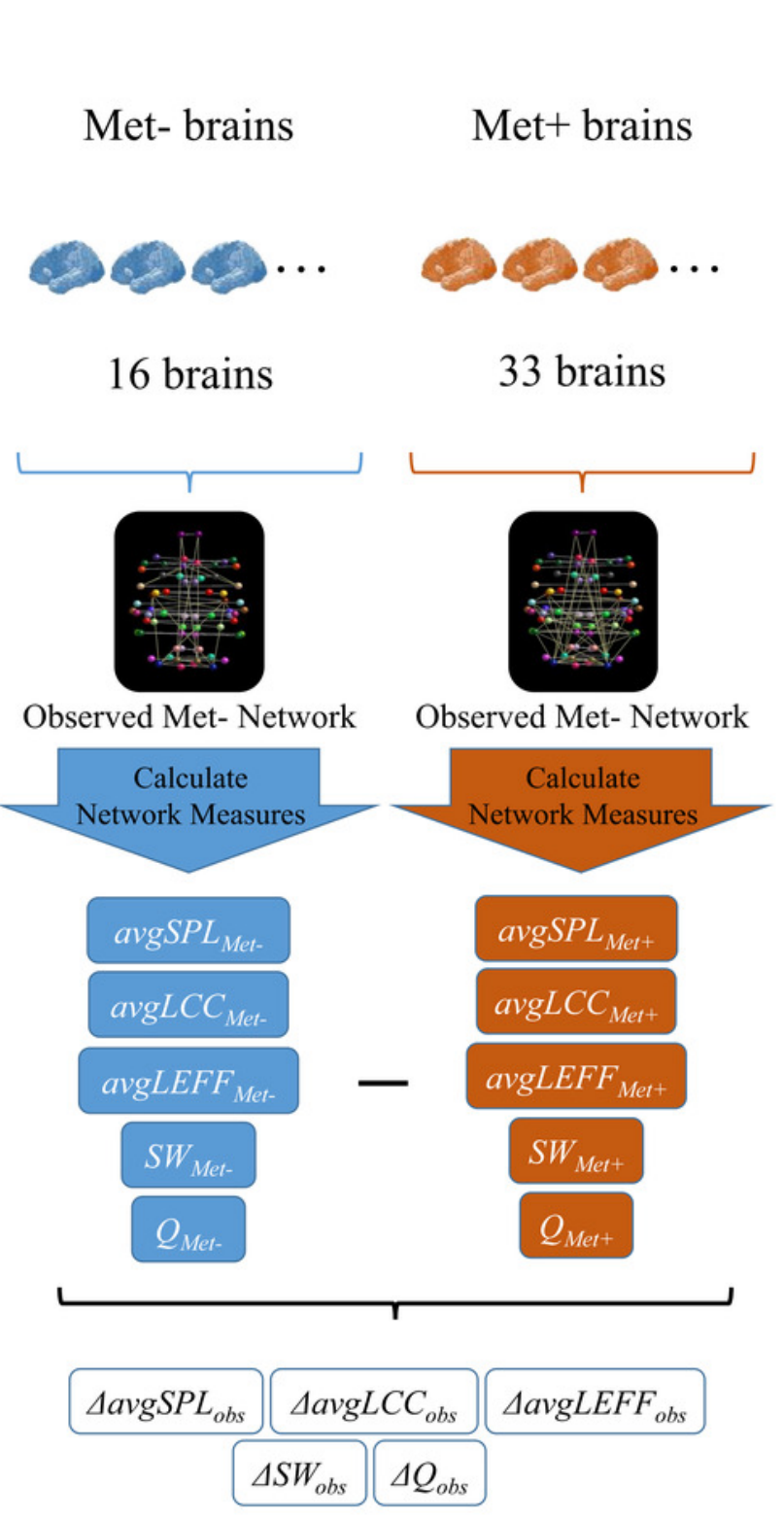

Observed Difference
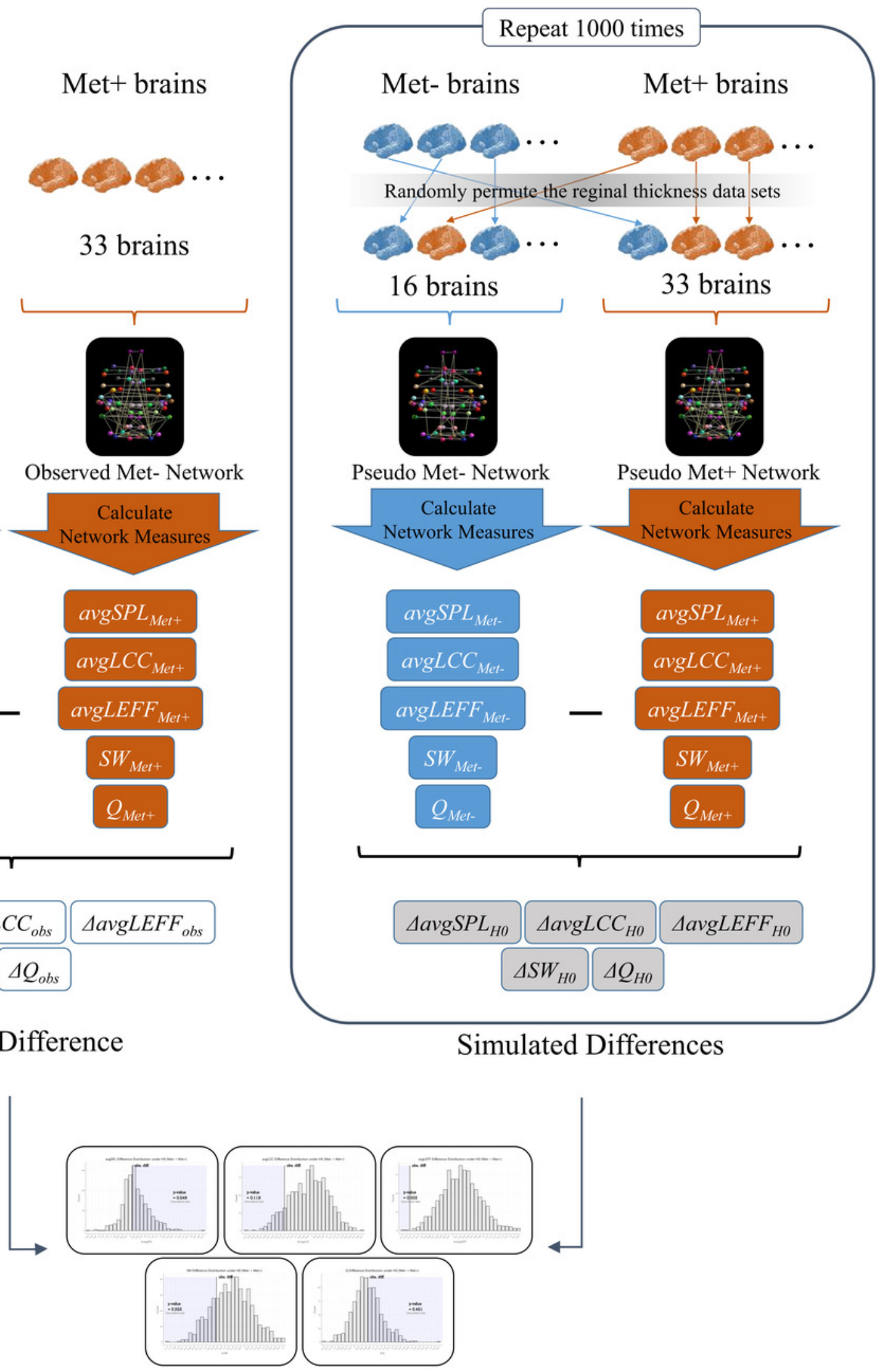

Calculate "p-values" from the distributions under null hypotheses 


\section{Figure 2}

Heat maps of the brain structural correlation network of the BDNF Val homozygotes (A) and Met carriers (B).

These heat maps represent each strength of inter-regional cortical thickness correlation of 68 brain regions. The color scale corresponds to the absolute values of Spearman's correlation coefficient. In a heat map of the brain network, grids represent sub-divided brain regions (i.e., modules). From these grid patterns, we observed that the Met carrier brain network had clearer module architecture than the Val homozygote brain network. When we focused on colors in each heat map, the Met carriers' map had more red areas whereas the Val homozygote heat map had more green areas. This observation suggested that the Val homozygote networks consisted of middle connections whereas the Met carrier networks had more connections with high strength. In both groups, each brain region had the strong correlation with the counterpart of the region; these correlations are described in the heat maps as the red lines running diagonally. The abbreviations of the brain regions used in these heat maps are summarized in Supplementary Table 1. 


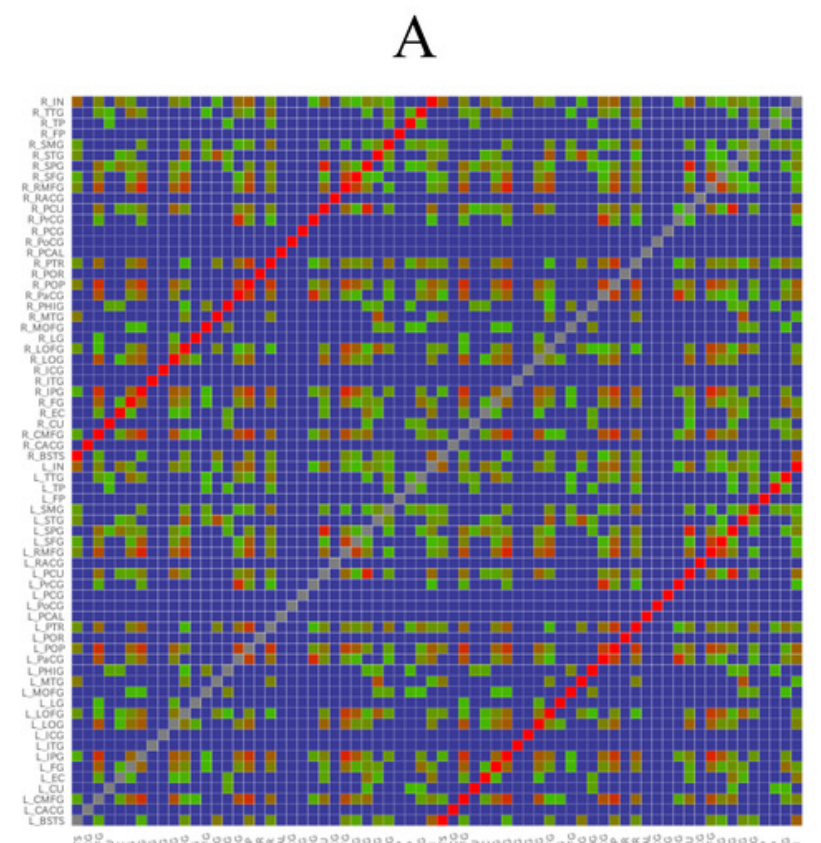

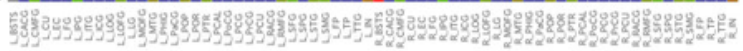

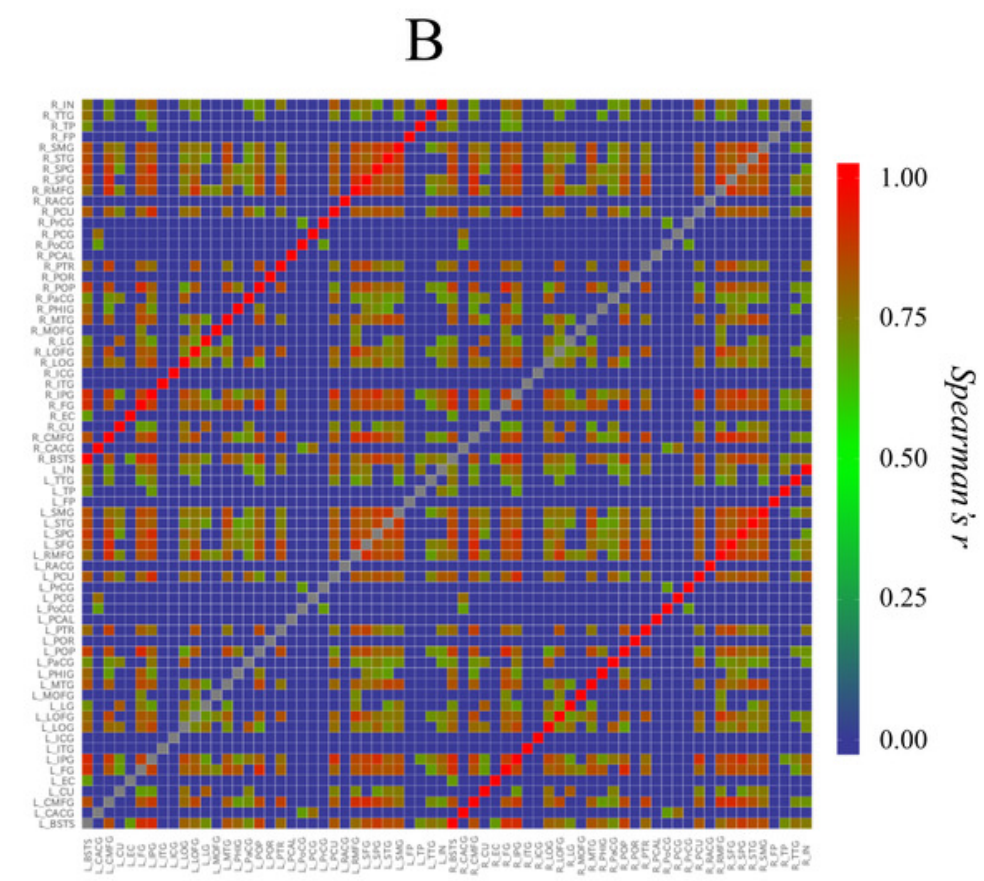


Figure 3

Circular graphs of brain structural correlation network of the BDNF Val homozygotes (A) and Met carriers (B).

These circular graphs are describing the inter-regional cortical thickness correlation, where the nodes represent brain regions, and the edges represent undirected connections between the regions. Each of the edge weight corresponds to the absolute value of Spearman's correlation coefficient. In these circular graphs, we only showed the 100 strongest connections. The Met carrier network had fewer brain regions related to the 100 strongest connections than the Val homozygote network. The abbreviations of the brain regions used in these circular graphs are summarized in Supplementary Table 1.

A

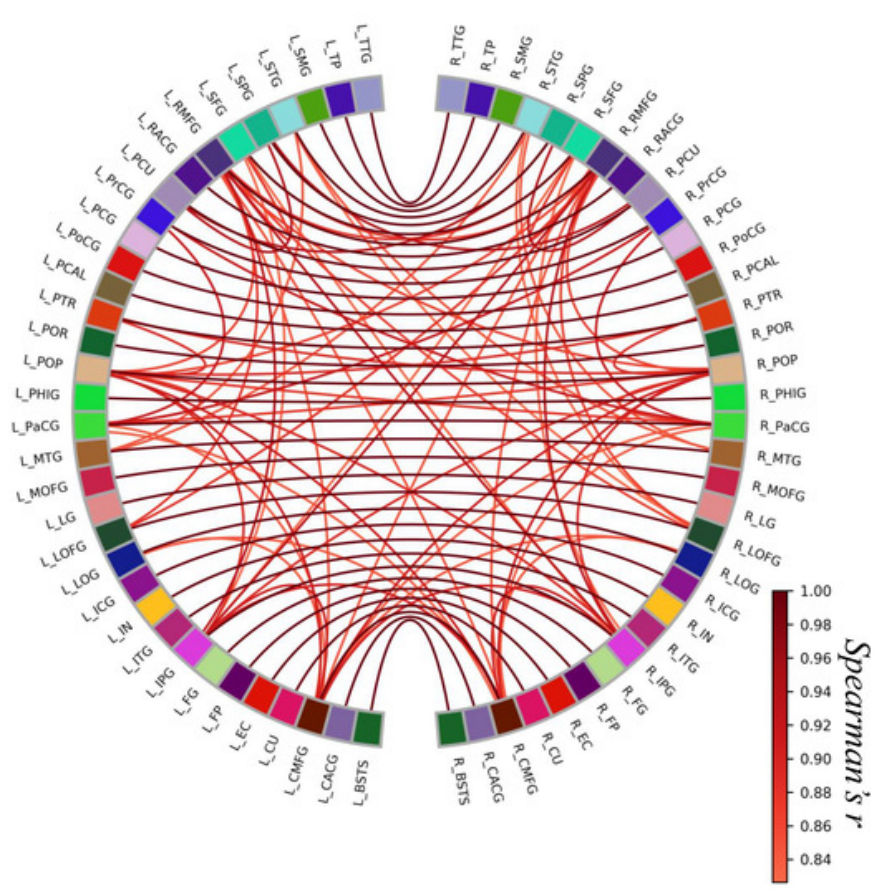

B

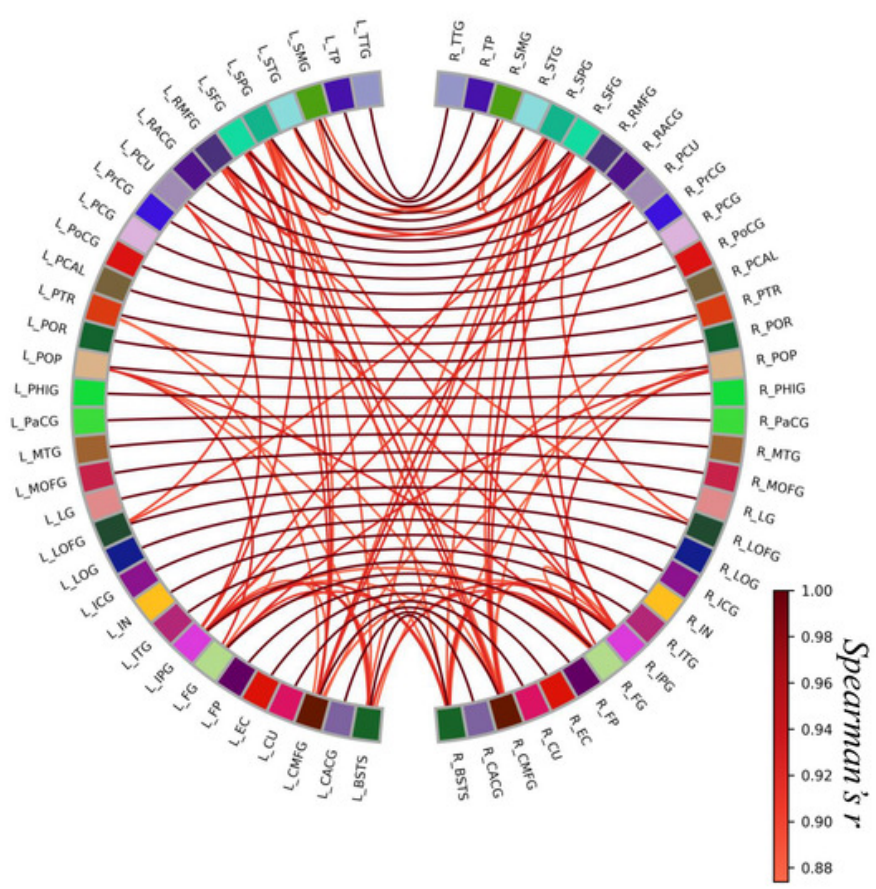




\section{Figure 4}

Permutation distributions and $p$-values of the network measures calculated from the structural correlation networks.

These histograms were obtained by repeating the calculation of the network measure difference between the randomly-partitioned groups of subjects. Each of the p-values was calculated as the frequency that the simulated differences were greater (or less) than each of the observed differences. The average local efficiency (avgLEFF) of the Met carriers' network was larger than that of the Val homozygotes' network, and the statistical difference between the avgLEFF between the two networks was significant ( $p$-value $=0.002)(C)$. There were no significant differences between the two groups in regard to the average shortest path length (avgSPL), the average local clustering coefficient (avgLCC), small-worldness (SW), and modularity $(Q)$ ( $p$-value $\geqq 0.01)(A, B, D$, and $E)$. 
(A)

Difference Distribution under Null Hypothesis (H0: Met- $=$ Met + )

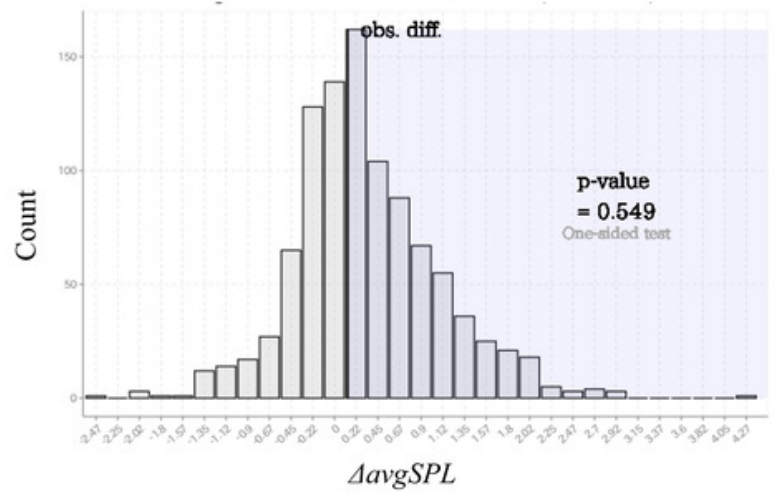

(C)

Difference Distribution under Null Hypothesis (H0: Met- $=$ Met + )

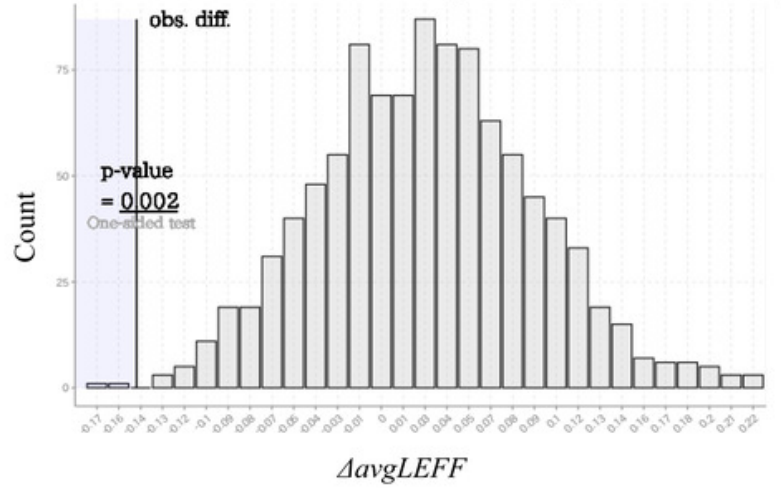

(E)

Difference Distribution under Null Hypothesis (H0: Met- $=$ Met + )

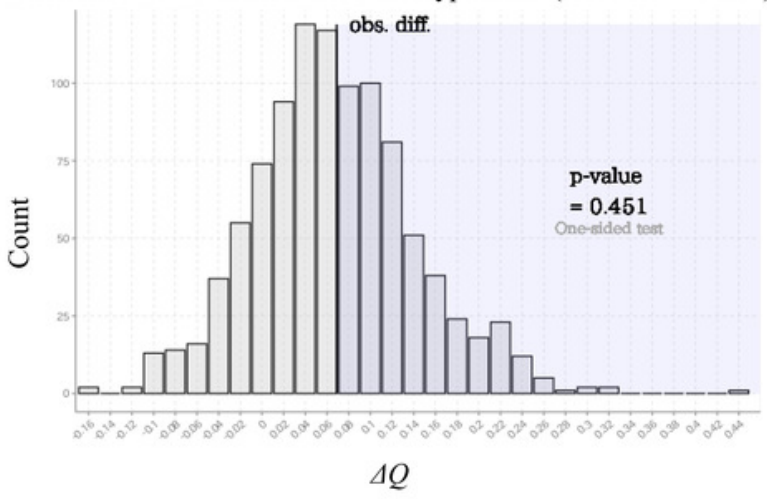

(B)

Difference Distribution under Null Hypothesis (H0: Met- = Met + )

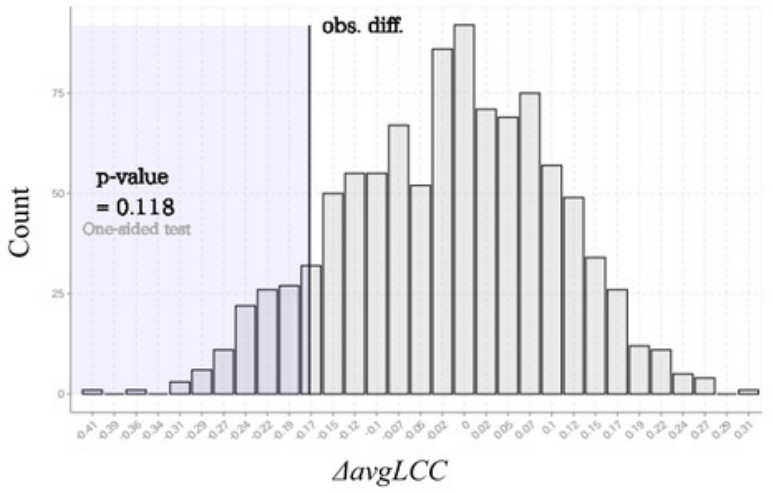

(D)

Difference Distribution under Null Hypothesis (H0: Met- $=$ Met + )

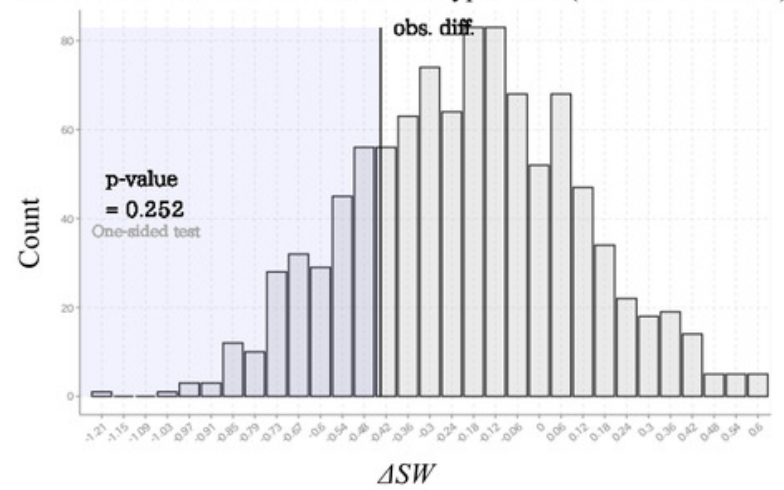




\section{Table 1 (on next page)}

Genotype frequency, gender, age, and years of education of the two groups.

Abbreviations: Val $=$ valine, Met $=$ methionine,$M=$ male; $F=$ female; $S D=$ standard deviation. 


\begin{tabular}{|c|c|c|}
\hline \multirow[b]{2}{*}{ Characteristics } & \multicolumn{2}{|c|}{ Genotype } \\
\hline & $\begin{array}{l}\text { Val homozygotes } \\
\qquad(n=16)\end{array}$ & $\begin{array}{l}\text { Met carriers } \\
\qquad(\mathrm{n}=33)\end{array}$ \\
\hline $\begin{array}{l}\text { Gender } \\
(M: F)\end{array}$ & $13: 3$ & $23: 10$ \\
\hline $\begin{array}{l}\text { Age [years] } \\
\text { (Wean } \pm \mathrm{SD})\end{array}$ & $40.5 \pm 11.3$ & $41.4 \pm 11.5$ \\
\hline $\begin{array}{c}\text { Years of education } \\
\text { (Wean } \pm \mathrm{SD})\end{array}$ & $16.4 \pm 3.0$ & $16.5 \pm 2.3$ \\
\hline
\end{tabular}




\section{Table 2 (on next page)}

The statistical comparison result regarding network measures of the brain structural correlation networks from the BDNF Val homozygotes and Met carriers.

The bold text with ' $*$ ' indicates significance ( $p$-value $<0.01$ ). Abbreviations: BDNF $=$ brainderived neurotrophic factor, Val $=$ valine, Met $=$ methionine . 


\begin{tabular}{|c|c|c|c|c|}
\hline Weighted Global Network Measure & Val Homozygotes & Met Carriers & p-value & \\
\hline $\begin{array}{l}\text { Average Shortest Path Length } \\
\text { (avgSFL) }\end{array}$ & 1.814 & 1.657 & 0.549 & \\
\hline $\begin{array}{l}\text { Average Local Clustering Coefficient } \\
\text { (avgLCC) }\end{array}$ & 0.551 & 0.715 & 0.118 & \\
\hline $\begin{array}{l}\text { Average Local Efficiency } \\
\text { (avgLEFF) }\end{array}$ & 0.484 & 0.627 & 0.002 & $*$ \\
\hline Small-wor ldness (SH) & 0.997 & 1.409 & 0.252 & \\
\hline Modularity (Q) & 0.190 & 0.119 & 0.451 & \\
\hline
\end{tabular}

